\title{
Aprendizagem e mecanismos de aprendizagem de trabalhadores em empresas da fruticultura irrigada do Baixo Médio São Francisco
}

\author{
Learning and learning mechanisms of employees of \\ irrigated fruit culture companies located in the \\ navigable waters of São Francisco river
}

\author{
Elisabeth Loiola ${ }^{1}$ \\ Marcos Emanoel Pereira² \\ Sônia Gondim²
}

\begin{abstract}
Resumo: O objetivo deste artigo é relacionar aprendizagens e mecanismos de aprendizagem de trabalhadores de empresas de fruticultura irrigada do Baixo Médio São Francisco (BMSF), para compreender como eles lidam com as mudanças da condição de trabalhadores de agricultura familiar para a de trabalhadores de agricultura empresarial, e com aquelas derivadas dos desafios competitivos das organizações para inserção em mercados internacionais. Para alcançá-lo, realizou-se uma pesquisa survey cross section, com amostra por acessibilidade, composta por 32 empresas e 950 trabalhadores. O segmento produtivo e a região foram escolhidos por ilustrarem situações paradigmáticas de mudanças em práticas tradicionais de produção e de organização do trabalho e também porque processos de aprendizagem de trabalhadores rurais ainda são pouco enfocados em pesquisas na área de administração. Verificou-se a ocorrência de aprendizagem individual - diferença entre o domínio atual sobre as tarefas de trabalho e o domínio inicial -, associada à utilização de poucos mecanismos informais e não estruturados de aprendizagem. Os resultados encontrados não validaram a primeira hipótese de que a aquisição de conhecimentos em situações de trabalho se associa à variedade, intensidade e interação dos mecanismos de aprendizagem. A segunda hipótese de que o uso de mecanismos de aprendizagem internos e externos se associa ao tipo e à quantidade de conhecimentos foi corroborada. O descompasso relativo entre resultados de pesquisas revisadas e os aqui relatados vincula-se a diferenças entre características das empresas, estruturas de mercado, produtos e organização do trabalho.
\end{abstract}

Palavras-chave: Aprendizagem no trabalho. Mudança organizacional. Mecanismos de aprendizagem. Fruticultura irrigada. Empresas rurais. Trabalhadores rurais.

\begin{abstract}
The general goal of this paper is to relate learning processes and learning mechanisms used by employees of irrigated fruit culture companies, located in the navigable waters of São Francisco river to understand how they deal with the changes in the working conditions from being family farmers and becoming corporate workers as well as those that result from competitive challenges of organizations to entry into international markets. Therefore, we conducted a cross section survey based on an accessibility sampling that consisted of 32 companies and 950 employees. This particular productive sector and region were chosen because they illustrate paradigmatic situations of changes in traditional production practices and work organization and also because rural workers'learning processes have been little explored in studies in the administration field. Individual learning was identified - difference between the current work experience and the previous experience - associated to the use of a few informal mechanisms instead of structured learning mechanisms. The results obtained did not validate the first hypothesis that the acquisition of work related knowledge is associated to variety, intensity, and interaction of learning mechanisms. On the other hand, the second hypothesis - that the internal and external learning mechanisms are associated to the amount of knowledge - was validated. The gap between the reviewed studies' results and those reported in this present study is related to the differences in companies' characteristics, market structures, products, and work organization.
\end{abstract}

Keywords: Learning at work. Organizational change. Learning mechanisms. Irrigated fruit culture. Rural enterprises. Rural workers.

\footnotetext{
${ }^{1}$ Universidade Federal da Bahia - UFBA, Ladeira do Acupe, 77/1103, Acupe de Brotas, CEP 40290160, Salvador - BA, Brasil, E-mail: beteloiola@oi.com.br

${ }^{2}$ Universidade Federal da Bahia - UFBA, Rua Rodrigo Argolo, 293, apto. 502, Rio Vermelho, CEP 41.940-220, Salvador - BA, Brasil, E-mails: emanoel@ufba.br, sggondim@terra.com.br
}

Recebido em 24/5/2010 — Aceito em 24/11/2010

Suporte financeiro: CNPq. 


\section{Introdução}

A crescente importância do tema aprendizagem organizacional no campo dos estudos organizacionais e da gestão está associada a mudanças nas organizações, mais evidentes a partir de meados da década de 1960 e início da década de 1970 . O conceito de aprendizagem, no entanto, ainda carece de maior precisão. Referindo-se a essa imprecisão, Pozo (2002, p. 59) diz que aprendizagem individual "é mais uma categoria natural que um conceito bem definido". Adicionalmente, observam-se lacunas de pesquisas na área de aprendizagem dos indivíduos em situações de trabalho. A combinação entre mudanças nos modelos de gestão e de organização do trabalho, imprecisões do conceito de aprendizagem e lacunas de pesquisa na área de aprendizagem dos indivíduos em situações de trabalho tem contribuído para o predomínio de uma visão de que a problemática da aprendizagem é cativa dos novos modelos de organização, negligenciando-se os padrões de aprendizagem das empresas tayloristas-fordistas.

Em meio a essas imprecisões, lacunas e negligências, a problemática da aprendizagem dos indivíduos no trabalho tem sido foco privilegiado de pesquisa nas áreas da Psicologia Organizacional e do Trabalho, e da Psicologia da Educação. Especialmente na primeira, tais pesquisas têm concorrido para uma melhor delimitação de tal fenômeno.

A vasta base teórica da psicologia tem contribuído para pesquisas desenvolvidas no campo dos estudos organizacionais sobre aprendizagem. No entanto, a utilização criteriosa dessa base teórica, elidindo-se os riscos de antropomorfização, mimetismo e reificação, tem sido um desafio que ainda não foi satisfatoriamente vencido. De fato, na área da administração, há uma zona de sombreamento entre aprendizagem organizacional e aprendizagem individual, uma tendência à redução da aprendizagem organizacional à aprendizagem de indivíduos nas organizações, em função do recurso à metáfora da aprendizagem individual e a crença difundida de que todas as organizações tendem a aprender da mesma forma, independentemente das singularidades de suas estruturas de mercado, de seus produtos, dos conhecimentos a eles vinculados, e das suas formas de organização do trabalho.

Sem ter a pretensão de enfrentar todos os desafios do campo da aprendizagem dos indivíduos em situações de trabalho, este artigo tem como objetivo relacionar aprendizagem e mecanismos de aprendizagem usados por trabalhadores de empresas da fruticultura irrigada do BMSF, para compreender como eles aprendem e lidam com as mudanças, tanto na transição da condição de trabalhadores de agricultura de subsistência e familiar para a de trabalhadores de agricultura empresarial quanto com as derivadas dos desafios competitivos enfrentados pelas organizações para se inserirem nos mercados internacionais.
As empresas da fruticultura irrigada do BMSF foram escolhidas como objeto de estudo por sua localização em região que passa por mudanças substantivas em suas práticas produtivas, substituindo a agricultura de subsistência pela agricultura empresarial, com uso intensivo de tecnologias. Pelo fato de a aprendizagem estar relacionada a mudanças, tais organizações são promissoras para o estudo de processos de aprendizagem. Além disso, processos de aprendizagem de trabalhadores rurais ainda são pouco explorados no campo da administração (BASTOS; GONDIM; LOIOLA, 2004; ANTONELLO; GODOY, 2009).

Além desta introdução, o artigo está estruturado em cinco seções, nas quais são discutidos os estados da arte e do campo da aprendizagem em situações de trabalho, descritos os procedimentos metodológicos, assim como discutidos os resultados. Na última seção, as principais conclusões, os limites e as contribuições da pesquisa são ressaltados, propondo-se uma agenda de novas pesquisas.

\section{Aprendizagem no trabalho}

A aprendizagem é um processo psicológico básico, amplo e complexo, relacionado a fatores intra e interpsíquicos, sociais e culturais por uma vasta literatura que $\mathrm{o}$ analisa de diferentes perspectivas teórico-metodológicas. A aprendizagem individual, na perspectiva cognitivista, é uma mudança atitudinal e comportamental relativamente permanente, associada à experiência, que envolve os planos afetivo, cognitivo e motor, garantindo a flexibilidade, adaptabilidade e capacidade transformadora do ser humano. Ela se relaciona, assim, a mudanças nas estruturas cognitivas e comportamentais dos indivíduos, com base em reflexão pessoal e em interação social. Nos processos de aprendizagem, os indivíduos adquirem Conhecimentos, Habilidades e Atitudes (CHAs) que podem ser inferidos a partir das mudanças atitudinais e comportamentais (ABBAD; BORGES-ANDRADE, 2004).

$\mathrm{O}$ conceito de aprendizagem individual envolve três dimensões: aquisição ou retenção, generalização e transferência. Realizando-se por meio de mecanismos internos e externos às organizações, formais e informais, a aquisição ou retenção do conhecimento de longo prazo relaciona-se ao tempo em que habilidades e comportamentos aprendidos permanecem usados no trabalho (TACLA; FIGUEIREDO, 2003; NERIS, 2005; LOIOLA; NERIS; BASTOS, 2006). A generalização consiste no grau em que conhecimentos, habilidades e atitudes aprendidos são aplicados a situações e condições diferentes daquelas em que foram adquiridos. Retenção e generalização são consideradas condições necessárias ao uso dos conhecimentos aprendidos no trabalho. Ao abarcar retenção e generalização, a transferência de 
conhecimento estende-se a atividades não equivalentes às anteriormente desempenhadas, ou diferentes daquelas vivenciadas no processo de aprendizagem (ABBAD; BORGES-ANDRADE, 2004).

Pesquisas sobre aprendizagem de adultos em situações de trabalho têm buscado respostas para algumas questões. Por que os indivíduos aprendem de forma diferente? Como os indivíduos aprendem no trabalho? Como eles organizam e gerenciam suas atividades de aprendizagem? Como as organizações podem dar suporte às atividades de aprendizagem de indivíduos e de grupos? Quais as implicações da aprendizagem para a organização?

Em relação à primeira questão-chave, evidências de pesquisa revelam que os indivíduos aprendem de forma diferente em função de características pessoais (idade, habilidades cognitivas, orientação para aprendizagem, motivação, autoeficácia orientada para tarefa, etc.) e de características contextuais (conteúdo, metodologia, clima de aprendizagem, suporte de supervisor e de colegas de trabalho, gestão da aprendizagem, etc.) (POZO, 2002). Nessas pesquisas, os contextos de aprendizagem (local, organizacional) e a própria experiência de aprendizagem funcionam como fontes de restrições ou de estímulos à aprendizagem individual e à sua transferência para o trabalho (LOIOLA; ROCHA, 2002). A capacidade de aprender também é influenciada pela congruência entre conhecimentos pré-existentes e o novo conhecimento (SONNENTAG; NIESSEN; OHLY, 2004; COHEN; LEVINTHAL, 1990).

A aprendizagem no trabalho, segunda questãochave, realiza-se mediante engajamento em programas formais de treinamento ou formas de aprendizagens espontâneas ou não estruturadas ou informais, como "aprender fazendo", "aprender usando", "aprender por interação", e de atividades internas e fora do contexto de trabalho (LOIOLA; ROCHA; RASKIN, 2002; SONNENTAG; NIESSEN; OHLY, 2004). Prestes Motta (2003), em trabalho pioneiro e que não segue a linha de abordagem hegemônica na área, discorrendo sobre a aprendizagem de indivíduos em situação de trabalho, resgata a relação entre aprendizagem e organização do trabalho, evidenciando que os processos de aprendizagem em empresas tayloristasfordistas foram muito simplificados em função de suas características de organização do trabalho - o parcelamento das tarefas, a supervisão direta, o posto fixo de trabalho e a esteira de produção, prevalecendo mecanismos informais e internos de aprendizagem.

Sonnentag, Nielssen e Ohly acrescentam que, apesar das relações positivas entre aprendizagem formal e informal, resultados positivos de treinamentos são insuficientemente conhecidos e os de aprendizagem informal são ainda menos estudados, embora já nas décadas de 1980 e 1990, Levitt e March (1988), Cohen e Levinthal (1990) e Simon (1996) tenham focados aspectos da aprendizagem informal e seus resultados. Levitt e March (1988) evidenciaram a insuficiência dos mecanismos informais de aprendizagem para aumentar a diversidade de competências das empresas, pré-condição para a inovação. Cohen e Levinthal (1990) e Simon (1996) acentuaram a importância dos mecanismos informais internos à empresa e das interações suscitadas por tais mecanismos para a aprendizagem. Nesses trabalhos não se encontram elementos que possam sugerir explicações para os mecanismos de aprendizagem adotados pelas organizações devido a singularidades dos tipos de produto, dos conhecimentos vinculados à produção, dos sistemas de produção e do tipo de organização do trabalho.

Antonelli (2002) introduz uma visão mais matizada sobre a relação entre aprendizagem e mecanismos de aprendizagem. De acordo com esse autor, a utilização de mecanismos de aprendizagem internos e externos varia em função do tipo de conhecimento. Anand et al. (2002) acrescentam que, além de variar em função do tipo de conhecimento, a utilização de mecanismos de aprendizagem formais e informais e internos e externos depende da quantidade de conhecimento que se quer adquirir. Tacla e Figueiredo (2003) e Figueiredo (2006) concluem que níveis mais avançados de aprendizagem tecnológica requerem maior variedade, intensidade de uso e interação entre diferentes mecanismos de aprendizagem, internos e externos às empresas e formais e informais.

Em relação à terceira questão-chave, os indivíduos gerenciam e organizam suas atividades mobilizando estratégias de aprendizagem de três tipos: cognitivas, que envolvem aspectos da reprodução, organização e elaboração da informação, subdividindo-se em reprodução, reflexão intrínseca, reflexão extrínseca; comportamentais, como a busca de ajuda interpessoal, em material escrito e sua aplicação prática; e autorregulatórias, que abarcam o controle da motivação, emoção e compreensão (WARR; ALLAN, 1998; PANTOJA, 2004; SONNENTAG; NIESSEN; OHLY, 2004). Quanto à quarta questão-chave, os principais suportes organizacionais à transferência da aprendizagem para o trabalho são a infraestrutura material (ferramentas), as oportunidades de aplicação, o estímulo a aplicar e o intervalo de tempo entre a aquisição da competência e a oportunidade de aplicação (quanto menor, melhor a transferência) (ABBAD; BORGES-ANDRADE, 2004). Por fim, quanto aos resultados para as organizações, quinta questão-chave de estudos na área, as pesquisas evidenciam que podem ser positivos ou negativos, de curto e de longo prazo (SONNENTAG; NIESSEN; OHLY, 2004).

Nesta pesquisa, o foco é a aprendizagem de tarefas pelos trabalhadores de empresas da fruticultura irrigada do BMSF. Em vista disso, a próxima seção 
apresenta uma discussão com base em bibliografia mais focada em pesquisas que investigam como os trabalhadores aprendem no trabalho e em autores que fornecem bases conceituais para essa investigação.

\section{Procedimentos metodológicos}

Esta seção descreve os procedimentos de método. Inicia-se com a problematização do objeto, a definição de problema de pesquisa e hipóteses. Em seguida, são apresentados objetivos, tipo de pesquisa, amostra, instrumento e sistemática de tratamento dos dados.

\subsection{Problematização, problema de pesquisa e hipótese}

Conforme descrição apresentada dos aspectos-chave do processo de aprendizagem de adultos em contextos de trabalho, a aquisição de conhecimentos relaciona-se à aprendizagem dos indivíduos em organizações, cujos mecanismos, internos, externos, formais e informais são viabilizados pela organização e utilizados pelos indivíduos em situações de trabalho ou fora dele (TACLA; FIGUEIREDO, 2003; NERIS, 2005; LOIOLA; NERIS; BASTOS, 2006). Mecanismos de aprendizagem são formas nas quais os indivíduos se engajam para aprender - eventos estruturados e não estruturados, realizados dentro ou fora da empresa.

Conforme já assinalado, Levitt e March (1988) veem vantagens na aprendizagem por meio dos mecanismos informais, enquanto Cohen e Levinthal (1990) afirmam que os mecanismos informais são insuficientes se o objetivo a alcançar é aumentar a diversidade de competências das empresas, o que permitiria a inovação. Já Simon (1996), além de enfatizar a importância dos mecanismos internos, coloca em relevo a interação entre os membros da organização com quem detenha o conhecimento a ser adquirido como requisito para a aprendizagem. As três referências tratam de forma polarizada os mecanismos de aprendizagem formal e informal, sem explorar possíveis benefícios advindos de sua utilização combinada.

Estudos mais recentes, todavia, não corroboram essa visão dicotômica dos mecanismos de aprendizagem. Para Antonelli (2002), por exemplo, a utilização de mecanismos internos e externos varia em função do tipo de conhecimento. Quanto maior a complexidade do conhecimento tecnológico requerido para gerar novas tecnologias, maior será a probabilidade de utilização de mecanismos externos de aprendizagem. Em sentido contrário, quanto maior a necessidade de conhecimentos específicos de produtos e processos de uma organização, maiores são os incentivos para a internalização do processo de geração de conhecimento. Anand et al. (2002), por sua vez, abordam essa questão de outra perspectiva, relacionando-a à quantidade e ao tipo de conhecimento.
Ao tomarem por base a natureza (explícita ou tácita) e o volume de conhecimento que se está buscando (grande ou pequeno), esses autores desenvolvem uma categorização de aquisição de conhecimentos, pondo em relevo também a intensidade e tempo das interações requeridas: i) pequenos volumes de conhecimento explícito podem ser obtidos por mecanismos informais que dispensam interações mais intensas; ii) pequenos volumes de conhecimento tácito exigem interações mais intensas, de curto prazo; iii) grandes volumes de conhecimento explícito, relacionados a informações rotineiras e facilmente compreensíveis, são adquiridos em treinamentos formais; e iv) grandes volumes de conhecimento tácito requerem interações pessoais estreitas e prolongadas.

Tacla e Figueiredo (2003), por seu turno, mostram a importância da variedade, intensidade e interação entre diferentes mecanismos de aprendizagem: formal e informal, externo e interno. Os autores realizaram estudo longitudinal (entre 1984 e 2000) sobre a utilização de mecanismos de aprendizagem dos trabalhadores da Kvaerner Pulping do Brasil, empresa de produção de bens de capital para o setor de celulose no Brasil. Evidenciaram que, na década de 1980, a empresa acumulou apenas competência de rotina na maioria das funções tecnológicas. Nessa década, ela se encontra em estágio inicial de domínio tecnológico, com pouca variedade e baixa intensidade de uso de mecanismos de aprendizagem, prevalecendo o aprender fazendo (mecanismo informal).

Na primeira metade da década de 1990, a empresa passou a desenvolver atividades inovadoras em equipamentos de processo.

\begin{abstract}
Neste período, a empresa foi capaz de construir e acumular competências inovadoras em gestão de projeto e engenharia de sistemas; além disso, avançou na construção de competência inovadora em equipamentos de processo (TACLA; FIGUEIREDO, 2003, p. 120).
\end{abstract}

Tacla e Figueiredo (2003) atribuem esse maior domínio tecnológico ao aumento da variedade e da intensidade de uso dos mecanismos de aprendizagem. Especialmente no período de 1996 a 2000, a utilização de mecanismos de aprendizagem avançou em variedade, intensidade e bom funcionamento, evidenciando que a gestão dos processos de aprendizagem teve papel fundamental na acumulação de competências tecnológicas (TACLA; FIGUEIREDO, 2003). Os autores concluíram que a acumulação de competências tecnológicas ocorreu com base em mecanismos de aprendizagem internos e externos, formais e informais, e que variações na intensidade de uso explicaram as mudanças de estágio de domínio tecnológico da empresa.

Figueiredo (2006) retomou o modelo de investigação adotado por Tacla e Figueiredo 
(2003), para pesquisar tipos e níveis de capacidade tecnológica em uma amostra de 18 dos principais institutos de pesquisa em Tecnologia de Informação e Comunicação (TIC) no Brasil. O autor encontrou uma variedade de mecanismos de aprendizagem, intra e interorganizacional, associados a diferentes níveis de domínio tecnológico. Na fase inicial de domínio tecnológico, os treinamentos externos foram os principais mecanismos adotados. No entanto, por terem sido planejados apenas para atender a demandas pontuais, os impactos desses treinamentos sobre o domínio tecnológico não foram potencializados. $\mathrm{Na}$ fase intermediária, eles continuaram a ser o principal mecanismo de aprendizagem, com a diferença de terem seguido um planejamento global, com base em levantamento de necessidades gerais. Na fase mais avançada, além do treinamento externo, foram utilizados outros mecanismos de aprendizagem: palestras e cursos relacionados a desenvolvimento de software e TIC; participação em congressos e seminários; e interação com clientes e parceiros para desenvolvimento de projetos. No mesmo estudo, o autor verificou maior utilização de mecanismos de aprendizagem intraorganizacionais na fase avançada de domínio tecnológico: desenvolvimento exploratório e aplicações de engenharia; participação de engenheiros recém-formados em projetos; pesquisa aplicada; design; treinamento interno em desenvolvimento de software; e desenvolvimento em engenharia de processos e projetos mediante experimentação e comparação de dados interlaboratoriais.

Figueiredo (2006), como Tacla e Figueiredo (2003), conclui que o avanço das organizações em capacitação tecnológica associou-se à variedade, intensidade de uso e interação entre mecanismos de aprendizagem e que as trajetórias são específicas às organizações.

Nessa breve menção a referências teórico-empíricas sobre mecanismos de aprendizagem no trabalho, três aspectos podem ser destacados. O primeiro é a ausência de estudos com população de trabalhadores rurais, conforme já destacado por Bastos, Gondim e Loiola (2004) e Antonello e Godoy ( 2009), o que justificaria a necessidade de investigar se os aspectos teóricos e resultados empíricos relativos à aprendizagem e mecanismos de aprendizagem de trabalhadores urbanos, da indústria e de serviços, se aplicariam a trabalhadores rurais. $\mathrm{O}$ segundo aspecto foi a controvérsia em relação à contribuição de mecanismos informais de aprendizagem (aprender fazendo, aprender com o supervisor, aprender com os colegas), e internos e externos, para o alcance de objetivos mais complexos de inovação e de eficiência produtiva, suscitada por Levitt e March (1988), Cohen e Levinthal (1990) e Simon (1996). O terceiro aspecto a ser destacado é que a aprendizagem no trabalho é um fenômeno complexo, que parece variar nas organizações e demandar formas de mensuração mais sensíveis à captura de especificidades contextuais, beneficiando-se tanto de mecanismos de aprendizagem formal quanto informal, internos ou externos, a depender também do tipo de conhecimento a ser adquirido, do produto e do sistema de produção (LOIOLA, ROCHA, 2002; ANTONELLI, 2002; ANAND et al., 2002; TACLA, FIGUEIREDO, 2003; FIGUEIREDO, 2006). Esses três aspectos justificam a realização deste estudo, que foi guiado pela seguinte questão: Como os trabalhadores das empresas de fruticultura irrigada do BMSF aprendem a realizar o seu trabalho?

As hipóteses relacionadas a esse problema, com suporte nos aspectos destacados no parágrafo anterior e nas tensões identificadas no campo, são:

- H1: A aquisição de conhecimentos em situações de trabalho se associa à variedade, intensidade e interação dos mecanismos de aprendizagem.

- H2: O uso de mecanismos de aprendizagem internos e externos se associa a tipo e quantidade de conhecimentos.

\subsection{Objetivos}

O objetivo geral da pesquisa é analisar aprendizagens e mecanismos de aprendizagem de trabalhadores de empresas da fruticultura irrigada do BMSF, para compreender como eles aprendem e lidam com as mudanças da condição de trabalhadores de agricultura de subsistência e familiar para a de trabalhadores de agricultura empresarial, bem como com aquelas derivadas dos desafios competitivos enfrentados pelas organizações do setor para inserção em mercados internacionais. Desse objetivo geral, derivam três objetivos específicos: descrever as transformações introduzidas nos processos de organização da produção, de gestão do trabalho e da aprendizagem; identificar os processos de aprendizagem; identificar os mecanismos de aquisição de conhecimento dos trabalhadores das referidas empresas.

\subsection{Tipo de pesquisa, amostra, instrumento e sistemática de tratamento dos dados}

Trata-se de uma pesquisa extensiva crosssection. O Censo Frutícola da CODEVASF (2002), referente ao ano de 2001, indica a existência de 1448 organizações, 221 localizadas no Projeto Curaçá, 31 no Projeto Mandacaru, 250 no Projeto Maniçoba, 29 no Projeto Tourão, 122 no projeto Bebedouro e 795 no Projeto Nilo Coelho. Desse total, foram selecionadas 43 empresas de médio e grande porte (acima de 21 ha de área total), nove no Projeto Curaçá, quatro no Maniçoba, e 30 no Projeto Nilo Coelho. Simultaneamente ao trabalho de aproximação com a população inicial da pesquisa, novas informações 
foram coletadas em outras fontes, em especial na Associação dos Produtores e Exportadores de Hortigranjeiros e Derivados do Vale do São Francisco (VALEXPORT). Ao final, a amostra foi constituída de 32 empresas e 950 trabalhadores, definida com base no critério de acessibilidade, em função de dificuldades na conformação do universo e da população e quanto ao acesso dos entrevistadores às empresas.

Os dados foram coletados em questionário preenchido durante entrevista individual com os trabalhadores e gestores. Tendo em vista o problema de pesquisa e objetivos deste artigo, serão analisadas apenas algumas perguntas. A primeira questão pedia ao entrevistado que enumerasse suas principais tarefas ao longo da jornada de trabalho e indicasse seu grau de domínio antes de ingressar na empresa e seu domínio atual, numa escala de 1 (pouco domínio) a 7 (total domínio). A segunda questão solicitava que indicasse como havia aprendido a realizar seu trabalho com alternativas de resposta que incluíam dois mecanismos de aprendizagem mais estruturados (cursos dentro e fora da empresa) e os sete restantes eram, comparativamente, menos estruturados, além de 3 externos (cursos realizados fora da empresa, treinamento de fornecedores e visitas a outras empresas) e os seis restantes internos. A escala variava de 1 (não aprendeu usando esse mecanismo) a 3 (aprendeu fortemente usando este mecanismo) (Quadro 1).

O questionário foi pré-testado in loco para adaptá-lo às características dos pesquisados. Os dados foram digitados e analisados pelo SPSS (Statistical Package for Social Sciences), utilizando-se técnicas de estatística descritiva e inferencial. Foram calculados os valores médios das variáveis em cada dimensão analisada (domínio das tarefas antes e depois - o que aprendeu - e mecanismos de aprendizagem - como aprendeu) e suas significâncias estatísticas. Também foram realizadas entrevistas abertas com trabalhadores e gestores sobre suas histórias de vida profissional e suas experiências e conhecimentos no trabalho.

\section{O agronegócio no Baixo Médio São Francisco}

A emergência do agronegócio no Baixo Médio São Francisco (BMSF) é fruto de articulação entre os setores público, privado e associações regionais de produtores. Até recentemente, as principais atividades econômicas nessa região eram a criação de gado e a agricultura de subsistência, limitadas pelas condições inóspitas do clima semiárido. A pecuária extensiva é poupadora de mão de obra e de baixa incorporação de tecnologias, associando-se a processos de concentração da terra, uma vez que requer vastas extensões para a produção. Nesse contexto, a aprendizagem ocorre de forma espontânea, essencialmente pelo aprender fazendo.

$\mathrm{Na}$ agricultura de subsistência, independentemente do regime de propriedade da terra, observa-se uma rotina de trabalho que envolve toda a família em atividades agropecuárias de pequeno porte, com baixa utilização de tecnologias, e com o fim principal de assegurar a subsistência familiar. O que não é consumido pelas famílias é canalizado para mercados locais, principalmente para aquisição de outros produtos. Os trabalhadores, nesse contexto, detêm um saber integral sobre o ciclo de produção: preparar a terra, semear, plantar, acompanhar o desenvolvimento da lavoura, colher, consumir e vender. Nesse caso, a aprendizagem implica o aprender fazendo, embora existam organismos de extensão rural que introduzem processos de aprendizagens mais induzidos, com base em experimentação, de orientação tecnicista (LISITA, 2006).

Com a construção de barragens hidrelétricas e a implantação de grandes projetos de irrigação, a estrutura socioeconômica da BMSF mudou radicalmente. A adoção de modernas tecnologias de produção e comercialização, com a introdução de culturas não tradicionais de alto valor comercial, consolidou o predomínio das relações capitalistas de produção e a geração de uma riqueza sem precedentes na região. Tais mudanças levaram alguns estudiosos a denominar o BMSF de "Pomar do Sertão" ou "Nova Califórnia" (BLOCH, 1996).

No Vale do São Francisco, a viticultura e a cultura da manga devem seus desempenhos, em grande parte, às condições edafoclimáticas da região, favoráveis a uma produção de qualidade todo o ano. Tal vantagem comparativa vem permitindo a ocupação de janelas de oportunidades deixadas por outras regiões produtoras e melhores preços no mercado. Os avanços tecnológicos, introduzidos por produtoresexportadores merecem, todavia, destaque, já que o mercado de frutas é, hoje, altamente competitivo

Quadro 1. Mecanismos de aprendizagem.

\begin{tabular}{|ll|}
\hline \multicolumn{1}{|c|}{ Como aprendeu: mecanismos envolvidos na aprendizagem } \\
\hline 1. Ajuda de colegas & 6. Cursos oferecidos pela empresa \\
2. Por conta própria (experiência) & 7. Cursos realizados fora da empresa \\
3. Orientação do chefe ou supervisor & 8. Treinamento de fornecedores \\
4. Grupos de trabalho, discussão, solução de problemas & 9. Visitas a outras empresas. \\
5. Leituras e estudo individual & \\
\hline
\end{tabular}


quanto à diferenciação do produto. Associam-se às novas tecnologias certificações PIF (Produção Integrada de Frutas), que implicam utilização mais racional de defensivos e de adubação química, além de irrigação de forma combinada com previsões meteorológicas, condições do solo e estágio dos cultivos. A adoção da Eurepgap (European Good Agricultural Pratice) permite viabilizar a participação dos produtores locais em mercados consumidores que possuem elevadas barreiras como o americano, o europeu e, recentemente, o japonês (HORTIFRUTI BRASIL, 2004).

A nova dinâmica do agronegócio no BMSF tem despertado interesse de pesquisadores de diversas áreas. Bloch (1996) aponta os seguintes aspectos abordados em estudos sobre a agricultura irrigada na região: crescimento econômico, populacional e de concentração da riqueza; importância da região como exportadora de produtos tropicais; modificações e adaptações na nova tecnologia agrícola; impactos sobre o meio ambiente. Este trabalho explora nova área de interesse: a aprendizagem dos trabalhadores.

\section{Organizações pesquisadas, seus trabalhadores e aprendizagens}

Nesta seção, caracterizam-se as empresas pesquisadas, os trabalhadores e seus processos e mecanismos de aprendizagem. As empresas foram classificadas conforme os critérios ano de fundação, área plantada, principais culturas e número de trabalhadores, sendo descritas suas formas de organização e controle da produção e do trabalho. Os trabalhadores foram caracterizados com base em idade, sexo, escolaridade, idade de início do trabalho, local de residência, dentre outros fatores. Os processos de aprendizagem foram organizados em categorias indutivamente criadas a partir da análise das questões abertas que solicitavam a enumeração das tarefas realizadas e das experiências de aprendizagem relatadas na entrevista, conforme especificado na seção 3.3. Os mecanismos de aprendizagem são apresentados conforme constam no questionário. Já as relações entre aprendizagens e mecanismos de aprendizagem foram obtidas com base em técnicas de estatística inferencial.

\subsection{Caracterização das empresas e dos trabalhadores pesquisados}

Na Tabela 1, observa-se que 93,3\% das empresas pesquisadas (aproximadamente 29) se instalaram na região entre 1981 e 2004, sendo que 50,0\% desse total estabeleceram-se entre 1997 e 2004 . No período de 1965 a1980, encontram-se apenas 6,7\% das organizações. Esses dados ilustram o caráter recente do agronegócio no Baixo Médio São Francisco. No que tange à área plantada, 42,9\% das empresas pesquisadas (aproximadamente 13) têm até 100 hectares, enquanto $57,1 \%$ têm entre 101 e 600 hectares (aproximadamente 18). Na faixa das maiores propriedades, entre 400 e 600 ha, encontram-se, apenas, $10,7 \%$ do total das empresas pesquisadas. Conforme já indicado só foram pesquisadas empresas de médio e grande porte.

Em relação às principais culturas, $41,4 \%$ das organizações produzem manga e uva (aproximadamente 13). A monocultura, entretanto, ainda é expressiva, visto que cerca de $38 \%$ das empresas pesquisadas (aproximadamente 12) cultivam apenas uva ou 10,5\%, manga. Quanto ao número de trabalhadores, 43,3\% das organizações (aproximadamente 13) possuem até $199,10,1 \%$, mais de 799 trabalhadores e 66,6\%, menos de 400.

Notam-se, paralelamente à consolidação da agricultura empresarial no BMSF, mudanças expressivas nas práticas de trabalho tradicionais. Nas empresas pesquisadas, observou-se grande especialização do trabalho, formação de turmas para o desenvolvimento de tarefas específicas em cada ciclo do processo produtivo das lavouras e o forte parcelamento do trabalho. Essa organização da produção e do trabalho, na qual é central o parcelamento e o controle, é similar àquela praticada em organizações tayloristas-fordistas. $\mathrm{Na}$ verdade, o recurso à fragmentação das tarefas mostrou-se eficaz para a rápida transformação da condição de trabalhadores rurais migrantes, inclusive crianças e mulheres, em trabalhadores de manufaturas urbanas, desde os primórdios do capitalismo industrial até meados da década de 1970, quando o taylorismofordismo entrou em crise no mundo. Com o advento do taylorismo-fordismo, o parcelamento do trabalho significou a redução das tarefas a unidades supersimplificadas, o que foi acompanhado pela forte redução dos esforços de aprendizagem (PRESTES MOTTA, 2003).

Nas packing-houses, não há muita diferença em relação ao padrão anterior. Uma packing-house clássica divide-se em três setores de produção: para a Europa, para os Estados Unidos e para o Japão. O que diferencia uma linha de produção da outra são os diferentes níveis de exigência dos mercados, sendo o Japão o mais exigente, seguido do EUA e da Europa. A packing-house lembra uma linha de montagem de automóvel, com esteiras que transportam os frutos ao longo dos sucessivos estágios de tratamento e preparação para embalar e pelas esteiras elevadas que transportam as embalagens ao longo da esteira de preparação do fruto. $\mathrm{O}$ trabalhador posiciona-se em posto fixo. Em períodos de safra, em cada setor fica um representante credenciado pelos clientes para assegurar e atestar que o controle de qualidade foi respeitado. 
Participaram da pesquisa 950 trabalhadores, dos quais, $10,9 \%$ possuíam até 20 anos $(\mathrm{n}=103), 60 \%$ até 30 anos $(n=570)$, e apenas $14,2 \%$ declararam ter mais de 40 anos. Trata-se, portanto, de mão de obra predominantemente jovem, com poucos anos de estudo formal: $4 \%$ declararam-se analfabetos, $3 \%$, alfabetizados e $56,2 \%(n=533)$ com ensino fundamental incompleto.

A maior parte deles viveu em roça, fazenda ou vila $(57,1 \%, \mathrm{n}=542)$, com atividades ligadas à agropecuária (71,5\% do total, $\mathrm{n}=679)$. Quase $50 \%$ $(n=475)$ residem no local atual há pelo menos 10 anos, enquanto a outra metade, há mais de 10 anos. Tais dados são consistentes com o fenômeno de migração da roça para as cidades e vilas de irrigação, assim como apontam uma forte experiência anterior em atividades de agropecuária, embora já apareçam casos de experiência anterior em serviços $(22,1 \%) \mathrm{e}$ em indústria $(2,0 \%)$.

Sobre as atividades realizadas, $65,3 \%(\mathrm{n}=620)$ atuam no campo, $16,1 \%$ em atividades técnicas, $8,5 \%$ em atividades administrativas e 10,0\% em atividades de packing house; 29,9\% são temporários, enquanto do conjunto dos fixos (70\% dos entrevistados, $\mathrm{n}=665)$, $82,13 \%$ trabalham na empresa há pelo menos 5 anos
( $\mathrm{n}=780$ ) e apenas $5,1 \%$, há mais de 9 anos; $35,5 \%$ declaram ser o emprego atual o primeiro com carteira assinada e $69,1 \%(n=656)$, o terceiro. Por fim, o conjunto de entrevistados atribui elevada importância ao trabalho (média de 6,42, em uma escala de 7) e está satisfeito com o trabalho desempenhado (média de 5,82, em uma escala de 7).

\subsection{Aprendizagem individual no trabalho}

Nesta seção, constam os resultados da aprendizagem individual e das correlações entre essas aprendizagens e os mecanismos de aprendizagem.

\subsubsection{Apresentação dos resultados}

Os dados da Tabela 2 sugerem ter havido um expressivo grau de aprendizagem. O critério para se chegar a essa conclusão foi a comparação das médias entre o domínio atual relatado pelo entrevistado e o domínio inicial, em uma escala de 1 a 7 . Para avaliar a significância estatística entre as médias, foi usado o teste $t$ para amostras emparelhadas. A Tabela 2 apresenta as diferenças estatisticamente significativas entre a aprendizagem atual e a inicial

Tabela 1. Características das empresas $(n=32)$.

\begin{tabular}{|c|c|c|c|}
\hline Características & $\%$ & Características & $\%$ \\
\hline Período de fundação das organizações & & Principais culturas & \\
\hline $1965-1972$ & 6,7 & Uva e manga & 41,4 \\
\hline $1973-1980$ & 0,0 & Uva & 37,9 \\
\hline $1981-1988$ & 16,7 & Manga & 10,5 \\
\hline 1989-1996 & 26,6 & Outros & 10,2 \\
\hline $1997-2004$ & 50,0 & Número de trabalhadores & \\
\hline Área plantada por faixa & & Até 199 & 43,3 \\
\hline Até 100 hectares & 42,9 & Entre 200 e 399 & 23,3 \\
\hline Entre 101 e 200 ha & 25,0 & Entre 400 e 599 & 13,3 \\
\hline Entre 201 e 300 ha & 14,3 & Entre 600 e 799 & 10,0 \\
\hline Entre 301 e 400 ha & 7,1 & Entre 800 e 999 & 3,4 \\
\hline Entre 401 e 500 ha & 3,6 & 1000 ou mais & 6,7 \\
\hline Entre 501 e 600 ha & 7,1 & & \\
\hline
\end{tabular}

Fonte: pesquisa de campo, 2005.

Tabela 2. Grau de aprendizagem.

\begin{tabular}{lcccc}
\hline \multicolumn{1}{c}{ Categorias* } & $\begin{array}{c}\text { Domínio atual } \\
\text { ( a) }\end{array}$ & $\begin{array}{c}\text { Domínio inicial } \\
\text { (b) }\end{array}$ & $\begin{array}{c}\text { Grau de aprendizagem } \\
\text { (a-b) }\end{array}$ & p \\
\hline Recursos humanos & 6,45 & 3,15 & 3,30 & $* *$ \\
Apoio administrativo & 6,41 & 3,45 & 2,96 & $* *$ \\
Programação, controle de produção & 6,46 & 3,03 & 3,43 & $* *$ \\
Tratos culturais & 6,34 & 3,22 & 3,12 & $* *$ \\
Controle de qualidade & 6,50 & 3,55 & 2,95 & $* *$ \\
Packing House & 6,50 & 3,13 & 3,37 & $* *$ \\
\hline
\end{tabular}

Teste t para amostras emparelhadas, $* * p<.001, n=950 ; *$ Categorias indutivamente criadas a partir da análise da questão aberta sobre tarefa. Fonte: elaboração própria com base em resultados de campo, 2005. 
para cada uma das categorias de aprendizagem, obtidas pela análise indutiva da questão aberta em que o entrevistado enumerava as tarefas que fazia e o quanto possuía de domínio sobre elas. Ao se compararem os domínios iniciais e os atuais, percebe-se que em todas as categorias a aprendizagem variou de 2,95 (para a categoria controle de qualidade) a 3,43 (para a categoria programação e controle de produção), apresentando significância estatística em todas elas.

Embora as categorias da Tabela 2 sejam abrangentes, cabe lembrar que elas foram construídas com base em evocações dos trabalhadores sobre suas tarefas em um dia de trabalho normal. A análise da resposta aberta sobre as tarefas, para criação das categorias de aprendizagem mostrou que os entrevistados evocam seu trabalho de forma fragmentada - "desbrotar", "pinicar", "passar cal”, "desfolhar" -, sugerindo não mais possuírem uma visão integral sobre o processo de produção, tão comum a agricultores de subsistência. Dessa forma, as categorias refletem aprendizagens em tarefas que são específicas a produtos e processos das empresas pesquisadas. A categoria "tratos culturais", por exemplo, abarca um conjunto de tarefas ligadas ao cultivo e colheita da manga e da uva. Já a categoria "packing house" compreende um conjunto de tarefas desde o momento em que os frutos colhidos são submetidos a uma primeira inspeção visual de qualidade no início da esteira, na qual são colocados para serem limpos, submetidos a sucessivas inspeções e tratamentos de qualidade, embalados e armazenados para posterior transporte. A categoria de "programação, controle de produção" envolve o pessoal técnico e a gerência, que lida com o sistema PIF. Nessa categoria estão também as tarefas de cabos ou chefes de turma. Por fim, na categoria "controle de qualidade", encontram-se os trabalhadores mais experientes. Esse controle é realizado preponderantemente de forma visual, mas envolve também a realização de alguns ensaios e testes, em respeito às normas Eurepgap.

Os dados relativos aos mecanismos de aprendizagem por categoria de aprendizagem encontram-se na Tabela 3. A significância estatística foi calculada com a aplicação do teste $t$ para uma única amostra, utilizando-se o valor 2 como critério. Para a categoria de aprendizagem "recursos humanos", apenas os mecanismos de aprendizagem "ajuda de colegas", "por conta própria" e "orientação de supervisor/ chefe" foram muito utilizados, com valores de $p$ estatisticamente significativos. Nos casos das categorias de aprendizagem "apoio administrativo" e "programação e controle da produção", novamente apenas os mecanismos de aprendizagem antes citados foram estatisticamente significativos; já em relação à categoria "tratos culturais", somente dois mecanismos de aprendizagem foram estatisticamente significativos e mais utilizados "ajuda de colegas" e "orientação de supervisores e chefes". Para a categoria de aprendizagem "controle de qualidade", o único mecanismo de aprendizagem muito usado e estatisticamente significativo foi "orientação de supervisores e chefes". Por último, quanto à "packing house", foram muito utilizados e estatisticamente significativos os mecanismos "ajuda de colegas", "por conta própria" e "orientação de supervisores e chefes".

Registre-se que todos os mecanismos de aprendizagem com uso estatisticamente significativo pertencem à categoria dos menos estruturados e internos à organização, verificando-se quase nenhuma variação. Isso vale tanto nos domínios de aprendizagem mais operacionais, como "tratos culturais" e "packing house", como em "recursos humanos", "apoio administrativo", "programação e controle da produção" e "controle de qualidade". Tais resultados sugerem que os processos de aprendizagem não são geridos numa ampla gama de variedade de mecanismos.

Tabela 3. Aprendizagens e mecanismos de aprendizagem.

\begin{tabular}{lcccccc}
\hline \multicolumn{1}{c}{$\begin{array}{c}\text { Mecanismos de } \\
\text { aprendizagem }\end{array}$} & $\begin{array}{c}\text { Recursos } \\
\text { humanos }\end{array}$ & $\begin{array}{c}\text { Apoio } \\
\text { administrativo }\end{array}$ & $\begin{array}{c}\text { Programação, } \\
\text { controle de produção }\end{array}$ & $\begin{array}{c}\text { Tratos } \\
\text { culturais }\end{array}$ & $\begin{array}{c}\text { Controle de } \\
\text { qualidade }\end{array}$ & $\begin{array}{c}\text { Packing } \\
\text { house }\end{array}$ \\
\hline Ajuda de colega & $\mathbf{2 , 2 6}$ & $\mathbf{2 , 5 2}$ & $\mathbf{2 , 3 1}$ & $\mathbf{2 , 1 0}$ & $\mathbf{2 , 2 8}$ & $\mathbf{2 , 2 3}$ \\
Por conta própria & $\mathbf{2 , 5 1}$ & $\mathbf{2 , 3 2}$ & $\mathbf{2 , 1 7}$ & $\mathbf{2 , 0 2}$ & $\mathbf{2 , 0 0}$ & $\mathbf{2 , 2 9}$ \\
Orientação do supervisor & $\mathbf{2 , 5 0}$ & $\mathbf{2 , 6 8}$ & $\mathbf{2 , 5 9}$ & $\mathbf{2 , 6 0}$ & $\mathbf{2 , 8 1}$ & $\mathbf{2 , 3 8}$ \\
Visitas a outras empresas & 1,24 & 1,16 & 1,14 & 1,08 & 1,16 & 1,06 \\
Grupos de trabalho e estudo & 1,64 & 1,94 & 1,38 & 1,33 & 1,41 & 1,26 \\
Leituras & 1,64 & 2,00 & 1,29 & 1,14 & 1,31 & 1,04 \\
Cursos internos & 1,47 & 1,80 & 1,26 & 1,16 & 1,34 & 1,12 \\
Cursos externos & 1,33 & 1,52 & 1,16 & 1,03 & 1,16 & 1,00 \\
Treinamento de fornecedores & 1,24 & 1,26 & 1,23 & 1,16 & 1,13 & 1,12 \\
\hline
\end{tabular}

Os valores grafados em negrito representam um $\mathrm{p}<.001$ e estatisticamente positivo em termos de muita utilização, enquanto os valores em itálico configuram um $\mathrm{p}<.05$, estatisticamente significativo em termos de muito baixa utilização; valores sem itálico e sem negrito foram não estatisticamente significativos. Fonte: elaboração própria com base em resultados de pesquisa de campo. 


\subsubsection{Discussão dos resultados}

Os dados apresentados na Tabela 2 demonstram que os trabalhadores das empresas pesquisadas aprenderam de forma significativa sobre suas atividades e tarefas em suas respectivas áreas de trabalho. Em todas as categorias formadas pela reunião de tarefas afins, as diferenças entre os níveis de domínio antes de começar a trabalhar na empresa e o atual foram estatisticamente significativos. É digno de nota que os trabalhadores já possuíam relativo domínio das tarefas antes de ingressar nas organizações, o que sugere haver relativa congruência entre conhecimentos e habilidades pré-existentes e conhecimentos e habilidades incorporados nas tarefas que passaram a realizar. Tal resultado é compatível com o fato já relatado de que a grande maioria dos pesquisados declarou que viveu na roça onde exercia atividades ligadas à agropecuária. Conforme revisão de literatura realizada, a capacidade de absorção de novos conhecimentos e habilidades é potencializada quando essa congruência se verifica (SONNENTAG; NIESSEN; OHLY, 2004, COHEN; LEVINTHAL, 1990).

Constata-se, no entanto, que os mecanismos de aprendizagem dos trabalhadores não variaram muito, com pouca ênfase em mecanismos mais estruturados e externos de aprendizagem (treinamentos, por exemplo) (vide Tabela 3). Os mecanismos mais frequentes foram: "ajuda de colega", "por conta própria" e "orientação do supervisor". Os mecanismos "ajuda de colegas" e "por conta própria" não foram estatisticamente significativos na categoria de aprendizagem controle de qualidade. Este último resultado pode ser explicado pelo fato de ensinar controle de qualidade exigir conhecimentos técnicos, os quais, no setor estudado, parecem ser apenas do domínio do supervisor. A estratégia de aprendizagem "por conta própria" também não foi estatisticamente significativa na categoria de tratos culturais. Em relação ao resultado de tratos culturais, a explicação pode ser a de que tais trabalhadores, embora tenham competências acumuladas com relativa congruência com o que fazem atualmente, estão envolvidos em aprendizagens de novas técnicas de cultivo, o que tem demandado a ajuda de colegas e supervisores mais experientes.

A "orientação do supervisor ou chefe" foi o mecanismo de aprendizagem mais usado pelos trabalhadores em todas as categorias de aprendizagem. Esse resultado revela a importância das orientações diretas do técnico, em atividades de produção agropecuária. A baixa escolaridade dos trabalhadoresaprendizes e a pouca importância dos processos formais de aprendizagem podem ser outra explicação para a grande utilização do mecanismo antes mencionado. Guarda relação também com a forma de organização e controle do trabalho: trabalhadores em grupos, chefiados por "um cabo de turma", sempre um trabalhador mais experiente.

Os resultados sinalizam que não há evidências para corroborar a hipótese de que a aprendizagem dos indivíduos nas organizações associou-se à variedade, à intensidade e à interação dos mecanismos de aprendizagem, conforme preconizado por Tacla e Figueiredo (2003) e Figueiredo (2006). Isso porque houve aprendizagem individual, mas não se observou a variedade esperada de mecanismos de aprendizagem: prevaleceram os de aprendizagem interna, de natureza menos estruturada (ajuda interpessoal, orientação do supervisor, etc.). Assim, pode-se concluir a favor da insuficiência de interação entre os diferentes tipos de mecanismos, à exceção dos mecanismos ajuda de colegas, por conta própria e orientação do supervisor ou chefe, que parecem estar associados, tendo em vista sua presença em quase todas as categorias de aprendizagem. Por meio desses mecanismos, as empresas desenvolvem mais capacitações em atividades nas quais já estão engajadas, potencializando a eficiência produtiva, o que é essencial para torná-las competitivas nos mercados a que atendem (LEVITT; MARCH, 1988). Apesar de serem importantes, esses conhecimentos não contribuem para aumentar a diversidade de competências das empresas, o que se torna crítico para a criação de algo que seja realmente novo (COHEN; LEVINTHAL, 1990).

Todavia a corroboração da segunda hipótese - o uso de mecanismos de aprendizagem internos e externos se associa ao tipo e à quantidade de conhecimentos parece emergir das evidências coletadas na pesquisa. Os conhecimentos aprendidos relacionam-se estreitamente às tarefas que são cotidianamente realizadas pelos pesquisados, conforme as proposições de Anand et al. (2002) sobre a relação entre tipo de conhecimento e mecanismos de aprendizagem. No caso dos trabalhadores pesquisados, verificou-se a aquisição de grande volume de conhecimento tácito por meio de interações pessoais estreitas e prolongadas. As evidências também corroboram as postulações de Antonelli (2002) sobre uma relação estreita entre grau de complexidade de conhecimento e tipo de mecanismo de aprendizagem interno e externo. No caso em estudo, a aquisição de conhecimentos ligados às tarefas cotidianas dos trabalhadores pesquisados se deu primordialmente pela ativação de mecanismos de aprendizagem internos às empresas.

Como explicar a não validação da primeira hipótese vis-à-vis a revisão de literatura? Como explicar, então, essa aparente contradição? Em linhas gerais, pode-se dizer que as diferenças associam-se ao fato de que os contextos setoriais, organizacionais e de aprendizagem são muito diferentes. Essas diferenças comparativas explicam, em parte, os resultados encontrados, de acordo com postulação de Loiola e Rocha (2002), já comentada neste trabalho. 
Detalhando os fatores contextuais que concorrem para a explicação das diferenças encontradas nos resultados em relação àqueles de Tacla e Figueiredo (2003) e Figueiredo (2006), retoma-se a discussão da organização do trabalho e aprendizagem, relação ainda pouco explorada na literatura atual sobre aprendizagem no trabalho. O recurso à fragmentação das tarefas vem sendo utilizado desde os primórdios do capitalismo industrial, tendo se mostrado eficaz para a rápida transformação da condição de trabalhadores rurais migrantes, inclusive crianças e mulheres, em trabalhadores de manufaturas urbanas. Com o advento do taylorismo-fordismo, a simplificação das tarefas avançou ainda mais, transformando-se em parcelamento do trabalho, o que simplifica, por sua vez, os esforços de aprendizagem (PRESTES MOTTA, 2003).

Da perspectiva da organização do trabalho, as empresas pesquisadas são tayloristas-fordistas. No campo, o trabalho é parcelado, o que se pode verificar nas descrições dos trabalhadores sobre suas tarefas em um dia de trabalho. As tarefas por eles indicadas compunham o rol de tratos culturais, mas foram evocadas de maneira fragmentada. Com a simplificação, a aprendizagem das tarefas não exige a ativação de mecanismos de aprendizagem muito variados e mais complexos, o que se mostra coerente com os princípios do taylorismo. Além disso, os grupos de trabalho operam sob supervisão constante, daí a importância do mecanismo de "aprender com o supervisor" num trabalho que exige baixa especialização, por envolver, preponderantemente, habilidades motoras e visuais. A prevalência de tais habilidades fica evidente com a presença marcante da mulher no campo de produção de uva, por possuir maior habilidade motora fina, ser mais cuidadosa e mais delicada no trato com o fruto; já na produção de manga prevalece o homem, mais alto e mais resistente a trabalhos pesados. Conforme se viu anteriormente, o sistema de trabalho nas packing houses também é caracterizado pelo parcelamento das tarefas, com postos de trabalho fixos enquanto produtos e embalagens circulam em esteiras rolantes, à semelhança das linhas de produção das empresas automotivas tayloristas-fordistas.

As empresas pesquisadas utilizam modernos sistemas de irrigação e produtivos (PIF). Nelas, atividades que envolvem conhecimentos técnicos e habilidades cognitivas são da estrita responsabilidade dos gerentes das fazendas ou de uma pequena equipe formada por técnicos agrícolas e engenheiros agrônomos. Muitas das empresas pesquisadas já possuem sistemas de certificações especiais exigidos por mercados particulares, sendo o mais difundido o Eurepgap, da União Europeia. Comparado com o tipo de agricultura anterior praticado na região, esse padrão é novo, radicalmente diferente, mas ainda é um padrão de produção taylorista-fordista, com processos de aprendizagem simplificados e operados por meio de poucos mecanismos de aquisição de conhecimentos mais convencionais.

Há outras justificativas para as diferenças encontradas entre os resultados das pesquisas revisadas e os resultados desta pesquisa. As organizações pesquisadas por Tacla e Figueiredo (2003) e por Figueiredo (2006) e as empresas da fruticultura irrigada são muito diferentes, em outros aspectos, não apenas na forma de organização do trabalho adotada. Elas pertencem ao setor industrial e de serviços, são intensivas em capital, tecnologia ou conhecimento, além de adotarem sistema de produção por projeto. Possuem mão de obra estável, não apresentando variações sazonais significativas. Desenvolvem sistematicamente esforços internos de P\&D\&E, talvez com mais ênfase em Engenharia. Além disso, foram pesquisados por meio de estudos de caso longitudinais.

As empresas pesquisadas são do setor agropecuário, intensivas em mão de obra, e seu contingente empregado varia sazonalmente. Lidam com produtos perecíveis, destinados ao consumo humano e, embora utilizem tecnologias atualizadas, não podem ser consideradas empresas intensivas em tecnologias, por não desenvolverem atividades internas de inovação, a menos daquelas limitadas à adaptação de cultivos bem aceitos por mercados estrangeiros, dentro das especificidades edafoclimáticas locais. Nesse caso, são poucos os trabalhadores envolvidos.

\section{Conclusões}

Em síntese, os resultados desta pesquisa indicaram: mudança substantiva nas práticas produtivas com impactos no aumento da capacidade de resposta da organização a mercados competitivos; aprendizagem substantiva em relação às novas práticas de trabalho; pouca variabilidade de mecanismos de aprendizagem utilizados, muito semelhantes àqueles de domínio dos agricultores de subsistência e difundidos pelas práticas extensionistas; tendência à fragmentação do conhecimento do trabalho. Esses resultados reforçam a percepção de que as trajetórias de aprendizagem são específicas a empresas, setores, produtos, formas de organização do trabalho e também aos contextos de aprendizagens.

As evidências sugerem que o processo de modernização instalado, da perspectiva da gestão da aprendizagem, é de natureza conservadora. Em oposição à visão integrada do processo de trabalho do agricultor de subsistência, encontraram-se relatos fortemente fragmentados sobre o processo de trabalho, sobretudo em relação aos tratos culturais. Mesmo que tais tarefas constituam uma etapa importante do processo produtivo estudado, os trabalhadores parecem não ter o domínio completo de todo o processo. Como as empresas pesquisadas têm conseguido ampliar 
suas bases mercadológicas, assegurando níveis de eficiência produtiva, pode-se, todavia, sugerir que os mecanismos de aprendizagem utilizados têm sido suficientes para transformar trabalhadores da agricultura familiar e de subsistência em trabalhadores da agricultura empresarial. Frente às especificidades edafoclimáticas da região e às técnicas de produção, elas são dotadas dos requisitos competitivos para o acesso a mercados consumidores exigentes. Não há evidências, no entanto, de que tais condições sejam sustentáveis no futuro. Considerando a história do taylorismo-fordismo, conjectura-se que a visão parcelizada do trabalho pode acarretar problemas com a força de trabalho, repercutindo certamente sobre seu desempenho e, consequentemente, sobre o desempenho competitivo das empresas.

Todo trabalho de pesquisa apresenta limites e riscos derivados não só das escolhas teórico-metodológicas dos pesquisadores, como da maior ou menor acurácia na implementação dessas decisões. Esta pesquisa não está imune a esses limites e riscos. Novos limites e riscos podem ter se apresentado pela falta de experiência dos entrevistados em participar de pesquisas, das dificuldades de adesão de empresas e trabalhadores, pelo difícil deslocamento até empresas e trabalhadores, e pelas condições adversas de aplicação dos questionários no próprio campo, submetendo entrevistados e entrevistadores a muito calor e sol, embora essa última condição seja também positiva por sua vinculação a situações reais de trabalho.

Apesar dos limites apontados, a pesquisa pôs em evidência a aprendizagem de trabalhadores rurais e seus mecanismos, concorrendo para reduzir lacuna apontada na literatura. Permitiu também aumentar a base documental sobre mecanismos de aprendizagem informais e suas relações com a aprendizagem de indivíduos em situações de trabalho, que é ainda muito pequena. Pôde-se verificar ainda que achados de pesquisas sobre aprendizagem de trabalhadores urbanos em indústrias e serviços não dão conta das especificidades das aprendizagens e seus mecanismos nas empresas rurais pesquisadas. Concluiu-se que os processos de aprendizagem das empresas pesquisadas são específicos, regendo-se por poucos mecanismos, internos e informais, relacionados às formas de organização do trabalho, além de outros fatores já mapeados na literatura - tipo de produto, tipo de conhecimento e setor. Tal fato corrobora a asserção de que ainda há pouca acumulação de conhecimento sobre aprendizagem e seus mecanismos para que se possa falar genericamente de processos de aprendizagem. $\mathrm{O}$ caminho a percorrer para mapear especificidades das aprendizagens em empresas e organizações ainda é longo e desafiador.

Do ponto de vista do método, esta pesquisa também oferece contribuições ao campo de estudo da aprendizagem, por focalizar microprocessos de aprendizagem, cuja importância foi definida pelo próprio pesquisado a partir de pergunta aberta, o que não é usual quando se trata de pesquisa survey. Essa opção amplificou os desafios da pesquisa, mas mostrou-se não trivial e profícua. Ainda quanto aos métodos, a pesquisa contribuiu na definição de escalas e itens que favoreceram a sua compreensão por um conjunto de trabalhadores de baixa escolaridade e do meio rural. Para as empresas, os resultados encontrados podem servir de base para promover uma melhor gestão dos processos de aprendizagem, considerando seus padrões competitivos, mudanças ambientais, formas de organização do trabalho, perecibilidade e destino ao consumo humano de seus produtos e relações benefício/custo. Sobre a difusão dos resultados, registra-se que todas as empresas participantes receberam um relatório com os resultados encontrados - posicionando-as em relação aos valores médios das variáveis - o que constitui uma boa prática, de devolução de resultados, ainda pouco utilizada e valorizada no Brasil.

Toda pesquisa é um trabalho incompleto, sujeito a limites, mas sinalizador de novos enquadramentos para o fenômeno pesquisado. Essa máxima se aplica a esta pesquisa, destacando-se dois novos desafios que merecem ser enfrentados: o primeiro é compreender as inconsistências entre o aumento e, simultaneamente, a fragmentação de conhecimentos dos indivíduos em situações de trabalho nas organizações pesquisadas; o segundo é compreender a fragmentação do conhecimento versus o crescimento de uso de tecnologias e a acumulação de capacitações pelas organizações, o que lhes vêm permitindo atender a exigências de mercados sofisticados no exterior.

\section{Referências}

ABBAD, G. S.; BORGES-ANDRADE, J. E. Aprendizagem humana em organizações de trabalho. In: ZANELLI, J. C.; BORGES-ANDRADE, J. E.; BASTOS, A. V. B. (Org.). Psicologia, organizações e trabalho no Brasil. Porto Alegre: Artmed, 2004. p. 237-275.

ANAND, V. C. et al. Capital social: explorando a rede de relações da empresa. Revista de Administração de Empresas, v. 42, n. 4, p. 57-71, 2002.

ANTONELLI, C. Economics of knowledge and the governance of commons knowledge. Revista Brasileira de Inovação, v. 1, n. 1, p. 29-48, 2002.

ANTONELLO, C. S.; GOGOY, A. S. Uma agenda brasileira para os estudos em aprendizagem organizacional. RAE, v. 49, n. 3, p. 266-281, 2009.

BASTOS, A. V. B.; GONDIM, S.; LOIOLA, E. Aprendizagem organizacional versus organizações que aprendem: características e desafios que cercam essas duas abordagens de pesquisa. RAUSP - Revista de Administração, v. 39, n. 3, p. 220-230, 2004.

BLOCH, D. As frutas amargas do velho Chico: irrigação e desenvolvimento no Vale do São Francisco. São Paulo: Livros da Terra/Oxfam, 1996. 
CODEVASF. Censo frutícola do nordeste brasileiro 2001. Brasília, 2002. 1 CD-ROM.

COHEN, W. M.; LEVINTHAL, D. A. Absorptive capacity: a new perspective on learning and innovation. Administrative Science Quartely, v. 35, p. 128-152, 1990.

FIGUEIREDO, P. Capacidade tecnológica e Inovação em Organizações de Serviços Intensivos em Conhecimento: evidências de institutos de pesquisa em tecnologias da Informação e da Comunicação (TICs) no Brasil. Revista de Inovação Brasileira, v. 5, n. 2, p. 403-454, 2006.

HORTIFRUTI BRASIL. Lucro Baixo em 2004. Hortifruti Brasil, dezembro de 2004, p.17, disponível em: www. cepea.esalq.usp.br/hfbrasil/edicoes/31/uva.pdf, acesso em 03 de abril de 2008.

LEVITT, B.; MARCH, J. A. Organizational learning. In: COHEN, M. D.; SPROULL, L. S. (Ed.). Organizational learning (Organization Science). California, London: Sage Production, 1998.

LISITA, F. O. Considerações sobre extensão rural no Brasil. 2006. Disponível em: <http://www.cpap. embrapa.br/publicacoes/online/ADM077>. Acesso em: 15 de jul. 2008.

LOIOLA, E.; NERIS, J. S.; BASTOS, A. V. B. Aprendizagem em organizações: mecanismos que articulam processos individuais e coletivos. In: BORGES-ANDRADE, J. et al. Treinamento, desenvolvimento e educação em organizações $\mathrm{e}$ trabalho - fundamentos para gestão de pessoas. Porto Alegre: ARTMED, 2006. p. 114-136.

LOIOLA, E.; ROCHA, M. C. F. Aprendizagem no processo de seleção de ferramenta CASE para o Estado da Bahia. Revista de Administração Contemporânea - RAC, v. 6, n. 2, p. 145-166, 2002.

LOIOLA, E.; ROCHA, M. C. F.; RASKIN, S. Microprocesso de aprendizagem: o caso da delimitação da metodologia para avaliação e seleção de ferramenta CASE para o Estado da Bahia. In: ENANPAD, 26, 2002, Salvador. Anais... p. 1-15.

NERIS, J. S. Microprocessos de aprendizagem em organizações do Baixo Médio São Francisco. Dissertação (Mestrado)-Universidade Federal da Bahia, Escola de Administração, 2005. 217 p.

PANTOJA, M. J. Estratégias de aprendizagem no trabalho e percepções de suporte à aprendizagem contínua: uma análise multinível. Tese (Doutorado)-Instituto de psicologia, Universidade de Brasília, 2004.

POZO, J. I. Aprendizes e mestres: a nova cultura da aprendizagem. Porto Alegre: Artmed, 2002.

PRESTES MOTTA, F. Aprendizagem e psicodinâmica organizacional. EAESP/FGV/NPP - Núcleo de Pesquisas e Publicações, 2003. 54 p. Relatório de Pesquisa N. 9/2003.

SIMON, H. A. Bounded rationality and organizational learning. In: COHEN, M. D.; SPROULL, L. S. (Ed.) Organizational learning (Organization Science). California, London: Sage Production, 1996.

SONNENTAG, S.; NIESSEN, C.; OHLY, S. Learning at Work: Training and Development. In: COOPER, C. L.; ROBERTSON, I. T. (Ed). International review of Industrial and organizational Psychology. Germany: John Wiley \& Sons, 2004. v. 19, chap. 8. Technical University of Braunschweig.

TACLA, C.; FIGUEIREDO, P. N. Processos de aprendizagem e acumulação de competências tecnológicas: evidências de uma empresa de bens de capital no Brasil. Revista de Administração Contemporânea, v. 7, n. 3, p. 101-126, 2003.

WARR, P.; ALLAN, C. Learning strategies and occupational training. Internacional Review of Industrial and Organizational Psychology, v. 13, p. 83-121, 1998. 